\title{
EFFECT OF CONDUCTIVITY IN CORROSION PROBLEM USING BOUNDARY ELEMENT METHOD AND GENETIC ALGORITHM
}

\author{
Mohammed Suleman Aldlemy ${ }^{1, *}$, Mohamed A. Gebril1 ${ }^{2}$, Farag I. Haider ${ }^{2}$, Ayad Omran Abdalla ${ }^{1}$ and \\ A. K. Ariffin ${ }^{3}$ \\ ${ }^{1}$ Department of Mechanical Engineering, College of Mechanical Engineering Technology, Benghazi, Libya. \\ ${ }^{2}$ Mechanical department, Engineering Faculty, Benghazi University, Benghazi-Libya. \\ ${ }^{3}$ Department of Mechanical and Materials Engineering, Faculty of Engineering and Built Environment, University \\ Kebangsaan Malaysia, 43600 Bangi, Selangor, Malaysia. \\ *Correspondence: maldlemy@ceb.edu.ly
}

\begin{abstract}
Boundary element method applications with inverse solution are used to apply the indirect analysis for modeling of corrosion problem. Laplace equation has been used to model the electrical potential in the electrolyte surface. In this paper a computer modeling has been developed to visualize the effect of conductivity value in corrosion problem. Genetic algorithm is used to create the conductivity value based on the mechanics of natural selection and genetics. The boundary element method is then calculating the potential value of the whole domain. FORTRAN and MATLAB program have been developed to calculate and visualize the potential distribution in the domain. Two-dimensional example problems are analyzed to demonstrate the application of the proposed boundary element modeling procedure.
\end{abstract}

Keywords: Corrosion modeling; boundary element method; genetic algorithm; conductivity.

\section{Introduction}

Corrosion is a natural phenomenon, which involves the study of the chemical, physical, metallurgical and mechanical properties of material [1]-[5]. Since the corrosion occurs when two different metals are electrically connected in a corrosive environment, the least noble of the two corrodes more rapidly than when unconnected. For example, the corrosion in soil is the destruction of material resulting from exposure and interaction with the soil environment. Corrosion in soil is a major concern, especially as much of the buried infrastructure is aging. Corrosion in soil is complex phenomenon, with a multiple of variable involved. Chemical reaction involving almost each of the existing elements is known to take place in soil, and many of these are not yet fully understood. Variation in soil properties and characteristic across three dimensions can have a major impact on corrosion of buried structure [6]-[8]. Corrosion monitoring is often needed to aid in the prevention system. The electrochemical technique has been used intensively in the field as nondestructive test for examining corrosion of the soil corrosion as well as for analyzing the phenomenon by direct measurement on the surface of the soil [6], [9]-[11].

Retarding either the anodic or cathodic reactions, the rate of corrosion can be reduced can prevent the corrosion. This can be achieved in several ways: first prevention it is coming by conditioning the metal, conditioning the corrosive environment and by the electrochemical control [8], [12], [13].

Recently, numerical analysis such as boundary element method (BEM) has been used intensively to solve corrosion problem. The purpose of this study is to apply the indirect analysis for modeling of the corrosion problem. Boundary element method is a powerful tool in computational analysis, used to obtain solutions to the partial differential equations of a variety of physical problems with well-defined boundary conditions. The differential equation, which is defined over the entire problem domain, is transformed into a surface integral equation over the surfaces that enclosed entirely the problem domain. [13]-[17]. The surface integral equation can then be solved by discrediting the surfaces into smaller regions boundary elements, a major advantage of the boundary element method over the finite element method is that the discrimination occurs only on the surfaces rather than over the entire domain, and the number of boundary elements required is generally a lot less than the number of finite elements required. BEM able to solve corrosion problem however need the optimization is solution by using the genetic algorithm. The genetic algorithm is stochastic techniques based on the mechanism of natural selection and natural genetic. Genetic algorithm is very suitable to be used to settle optimization complex problem that is difficult settled by using the normal method, such as finite element method [18]. 
A mention in previous introduction corrosion problem is very common problem, therefore besides measurement work, it important to make corrosion simulation. BEM is a powerful tool in computational analysis especially for corrosion analysis to obtain solutions of corrosion problem with well-defined boundary conditions. BEM can easily accommodate geometrically complex boundaries, less unwanted information and less computing time. The genetic algorithm is used to optimize the conductivity based on the mechanics of natural selection and natural genetics. Measurement method is often difficult because the corrosion is inside the concrete, under the ground and under the sea. The study can be applied underground and under the sea. The purpose of this study is to apply the indirect analysis for modeling of the corrosion problem inside concrete, under the ground and under the sea by using boundary element method and genetic algorithm.

\section{Mathematic model and boundary conditions}

Assume that the material, which will be analyzed is in homogeneous electrolyte environment and the entire electrolyte environment is domain, $\Omega$, which surrounded by the boundary $\Gamma=\Gamma 1+\Gamma 2+\Gamma 3 \mathrm{a}+\Gamma 3 \mathrm{c}$ that shows in Figure 1 and Figure 2. Then, if assume that there is no addition or reduction of electric ion (ion accumulation) inside the electrolyte environment, the relationship between the current density and potential inside the electrolyte environment follows the Laplace equation [19]-[21]:

$$
\nabla^{2} \varphi=0
$$

where $\nabla$ is written using square coordinate $(x, y, z)$ :

$$
\nabla^{2}=\frac{\partial^{2}}{\partial x^{2}}+\frac{\partial^{2}}{\partial y^{2}}+\frac{\partial^{2}}{\partial z^{2}}
$$

with the boundary condition, this is related to that equation can be written as follows:

$$
\begin{gathered}
\phi=\phi_{\mathrm{o}} \operatorname{In} \Gamma_{1} \\
i=i_{a} \operatorname{In} \Gamma_{2} \\
\phi=-\mathrm{f}_{\mathrm{a}}(\mathrm{i}) \operatorname{In} \Gamma_{3 \mathrm{a}} \\
\phi=-\mathrm{f}_{\mathrm{c}}(\mathrm{i}) \operatorname{In} \Gamma_{3 \mathrm{c}}
\end{gathered}
$$

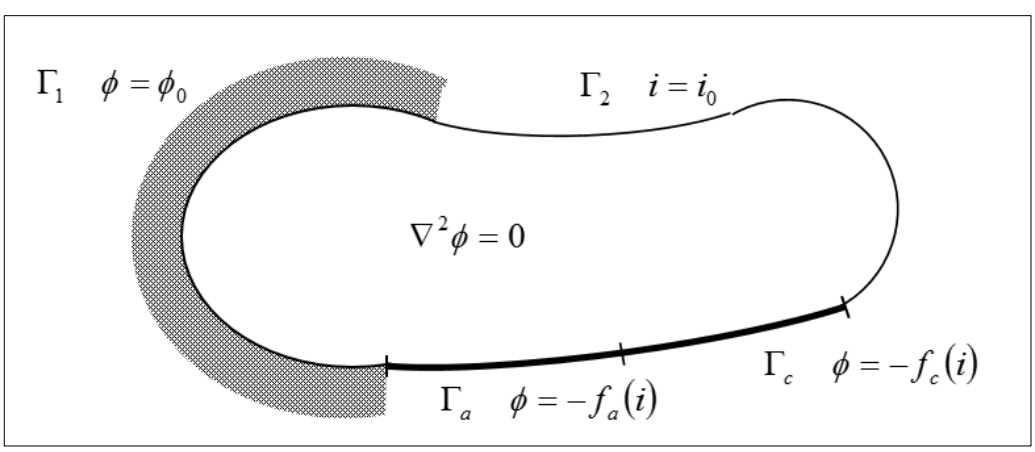

Figure 1: The basic equation and boundary condition for corrosion.

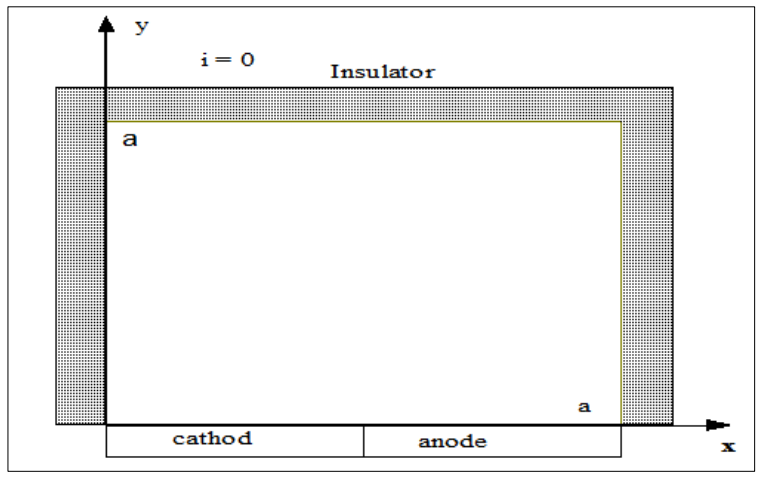

Figure 2: Tow-dimensional model of galvanic corrosion. 
where $I=-\kappa^{\phi \partial} / \partial n=i_{o}$, shows the current density, $\kappa$ is the conduction of electrolyte environment, $n$ is the outward normal from the boundary $\Gamma$, where $\Gamma_{1}$ is the known potential boundary or it is also called the essential condition or dirichlet Boundary. $\Gamma_{2}$ Is the known current density $(i)$ or $i$ is the derivative of $\phi$ and it is also called the natural condition or Newman boundary. $\Gamma_{3 \mathrm{a}}$ is the boundary on the corrode surface material (anode area) and $\Gamma_{3 c}$ is the boundary on the uncorroded surface material (cathode area). The sign minus on the right side of Equations 4 and 5 are the potential on the area near metal surface that the value is equal to the sign minus from the potential difference between metal and reference electrode like Saturated Calomel Electrode (SCE). In order to reduce the error on the Equation 1 if the exact value (even though it is unknown) from the potential and the current density, which is replace by the approximation equation and can be minimized by orthogonal zing the weight function, $\phi^{*}$ with the derivation on the boundary $i^{*}=\frac{\partial \varphi^{*}}{\partial n}$.

$$
\int_{\Omega}\left(\nabla^{2} \varphi\right) \varphi^{\bullet} d \Omega=\int_{\Gamma}\left(i-i_{o}\right) \varphi^{\bullet} d \Gamma-\int_{\Gamma}\left(\varphi-\varphi_{o}\right) i^{\bullet} d \Gamma
$$

where,

$$
i=\frac{\partial \varphi}{\partial n} \text { and } i^{*}=\frac{\partial \varphi^{*}}{\partial n}
$$

By integrating the left side of equation, it can be obtained as follows:

$$
\int_{\Omega}\left\{\frac{\partial \varphi}{\partial x_{k}} \frac{\partial \varphi^{\bullet}}{\partial x_{x}} d \Omega=\int_{\Gamma_{2}}-i \varphi^{\bullet} d \Gamma-\int_{\Gamma_{1}} i \varphi^{\bullet} d \Gamma+\int_{\Gamma_{2}} \varphi i^{\bullet} d \Gamma+\int_{\Gamma_{1}} \varphi i^{\bullet} d \Gamma\right.
$$

where $k=1,2,3$ and it is called the last Einstein's summation for used repetition index.

$$
\int_{\Omega}\left(\nabla^{2} \varphi^{\bullet}\right) \varphi d \Omega=\int_{\Gamma_{2}}-i \varphi^{\bullet} d \Gamma-\int_{\Gamma_{1}} i \varphi^{\bullet} d \Gamma+\int_{\Gamma_{2}} \varphi i^{\bullet} d \Gamma+\int_{\Gamma_{1}} \varphi i^{\bullet} d \Gamma
$$

The boundary integral equation (Equation 2) is the most used equation as the starting point from the boundary element method.

\section{Computer code for corrosion problem}

The theory mentioned from previous section will be employed to product a simple computer code written in FORTRAN for solving Laplace type problems. This code is valid for isotropic material and uses constant elements, which uses genetic algorithm (GA) with boundary element method (BEM) for 2D axisymmetric in potential analysis and corrosion problem. The program is simulation program, which makes use of the boundary element method instead of the normal finite element method; this can result in large productivity [22]. It can also be used for the simulation of general galvanic problems where any number of electrolytes and materials can be modeled. It can be used to study any electrostatic problem and can also be applied to stray current corrosion problems, with this program only need to define the elements on the boundary on surface.

The post processor has been designed to allow having an easy access to the large number of results produced by the parallel parametric study. By using this post-processor able to plot the potential distribution in the domain, it is created by using MATLAB program it is possible to plot any output quantity against the varying parameters. Post-processor generates since the information at the geometry is important to capture the details of corrosion problem behavior, by this post-processing.

\section{Results and discussion}

This paper presents the findings and describes each one in detail performed in FORTRAN program. Boundary element method is compute to solve the Laplace's Equation hence potential on the whole surface of the model. The model was performed in GA with BEM program written in FORTRAN for solving Laplace type problems to authenticate the accuracy and the dependability of the BEM by using FORTRAN program. Comparison study was conducted with the results based on the BEASY analysis software. The types of boundary conditions chosen for the comparison consider the two-dimensional problem of a galvanic corrosion. In this case $a=2 w$ and concede that the anode pleased in the medial of the galvanic couple area. The electrode is discretized into 16 constant elements such as pipelines and offshore structures are surrounded by a vast medium. It is appropriate to treat the corrosion problem. Figure 3 shows the potential distributions in the internal points by using BEM and BEASY. The figure shows a good agreement between both software's. 


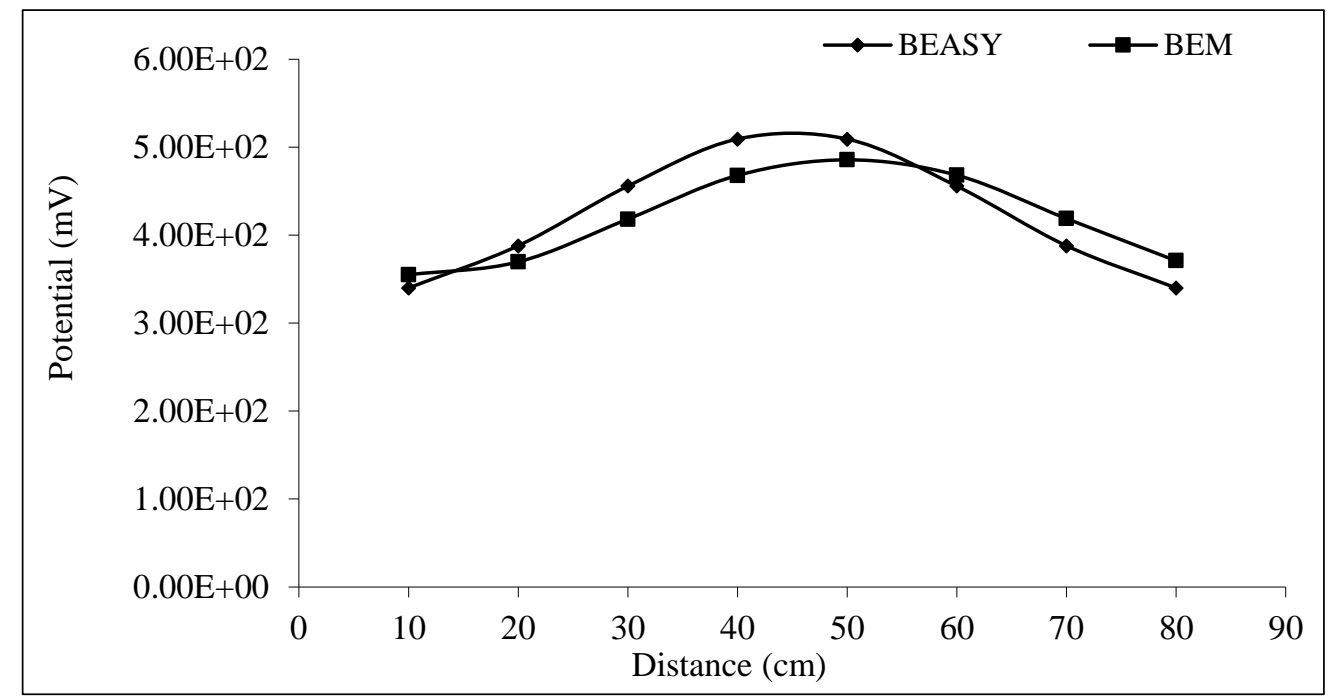

Figure 3: Potential distributions along the internal points using BEM and BEASY.

The types of boundary conditions chosen for the study is commonly used by researchers [22] consider the two-dimensional problem of a galvanic corrosion, as shown in Figure 4. In this case, the electrolyte is bounded by insulators at $x=0, x=a$ and $z=w, a=12 \mathrm{~cm}$ and $w=2 \mathrm{~cm}$.

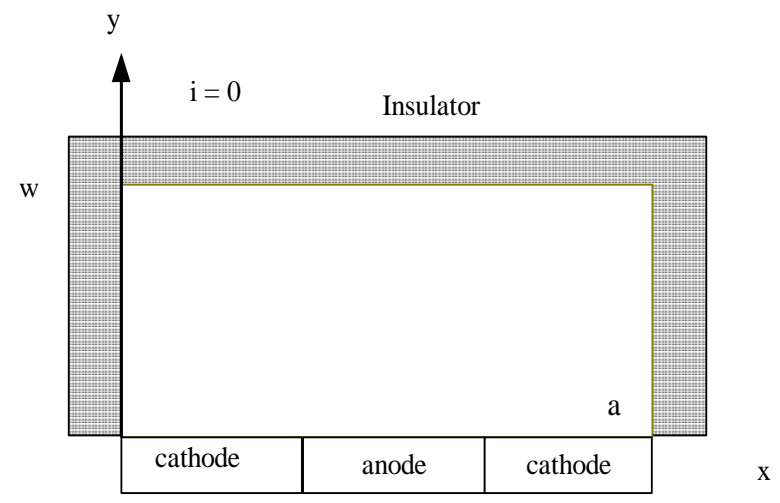

Figure 4: Two-dimensional models of galvanic corrosion couple.

Consider the two-dimensional problem of galvanic corrosion, in each models used the zinc material as anode and the steel material for the cathode. The potential values for steel $-810 \mathrm{mV}$ and for zinc -1101.2656 $\mathrm{mV}$ with same current density. The potential value is taken from BEASY document by select the current density of the zinc and steel to be closed, in order to make electrolyte reaction. From Figure 5 note that the rate of corrosion, proportional to the anodic, it can be estimated the corrosion area between the 75 and 80 along of $x$ axis because effect of polarization value of potential for zinc higher than steel. The cathodic current generated by the sacrificial anode and the cathode to be protected.

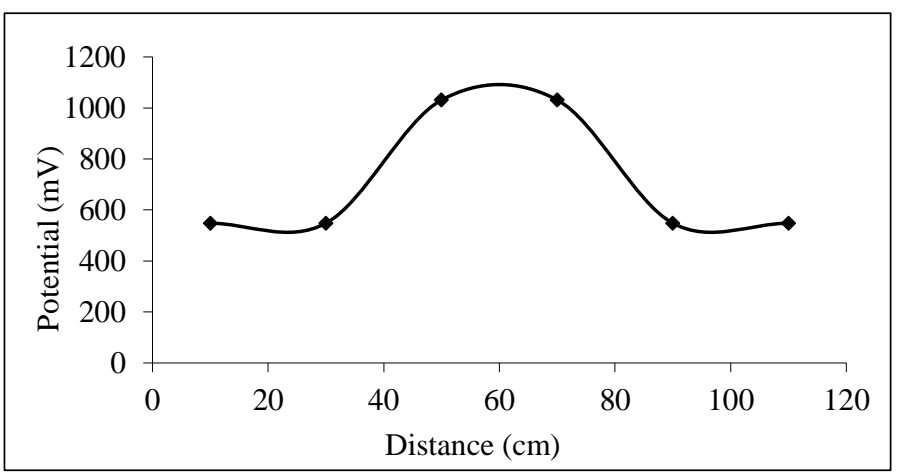

Figure 5: Potential distributions along of surface.

Page 4 of 6 
Figure 6 shows effect of conductivity value for potential distribution on the electrolyte surface for this model. The characteristic of corrosion surface can be described easily form the graph. When the conductivity is increasing the potential value in the internal point and at the surface electrolyte also increase. From figure indicate the larger conductivity value is 0.9302 give more effect to potential distribution compare with another value. It is indicated that there is a slight effect of corrosion when the values of conductivity decrease. The different potential values between each line are due to the effect of different conductivity. The larger conductivity value given a bigger change for potential value; potential value is larger if the conductivity is large. Those phenomenon shows that the large conductivity gives the effect for corrosion reaction. The important parameter that affected for potential distribution on the surface of electrolyte is conductivity value.

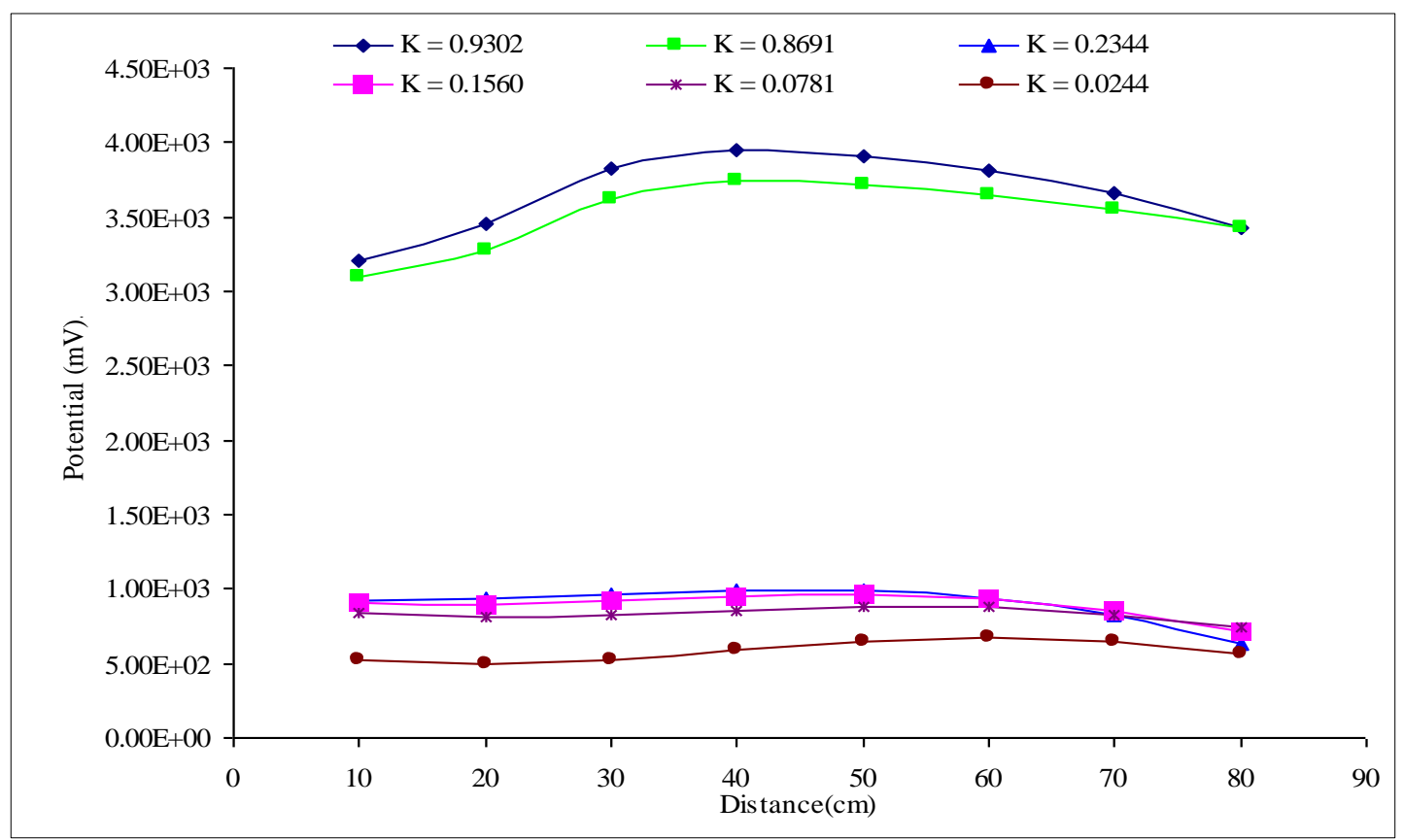

Figure 6: Effect of conductivity for Potential distribution.

\section{Conclusions}

The corrosion problem was identified using the boundary element method and genetic algorithm. In this study, the effects of conductivity was analyzed. The characteristic of corrosion can be described from potential distribution value on the surface of electrolyte. The boundary element method using FORTRAN program was employed to solve the Laplace's equation, hence the potential and on the whole surface of the domain can be determined, two dimensional example problems are given to demonstrate the effectiveness of the proposed boundary element modeling procedure. The conductivity value is used to give the effect for potential distribution. The results show that when the conductivity is increased the potential value in the internal point and at the surface electrolyte also increased. It also that no effect of corrosion when the value of conductivity decreased. Therefore, the important parameter that affect the potential distribution on the surface of electrolyte is conductivity

Conflicts of Interest: The authors declare no conflict of interest.

\section{References}

[1] K. R. Trethewey and J. Chamberlain, "Corrosion for Science and Engineering”. 2nd Edn. Longman. UK," 1995.

[2] M. A. Gebril, M. S. Aldlemey, F. I. Haider, and N. Ali, "Effect of Austenizing and Tempering Time on Corrosion Rate of Austenitic Stainless Steel in Oxalic Acid," vol. 980, pp. 46-51, 2014.

[3] M. A. Gebril, M. S. Aldlemey, and F. I. Haider, The effect of heat treatment of gray and nodular cast iron with ferrite matrix on mechanical properties and corrosion rate compared with medium carbon steel, vol. 936. 2014.

[4] D. Dwivedi, K. Lepková, and T. Becker, "Carbon steel corrosion: a review of key surface properties and characterization methods," RSC Adv., vol. 7, no. 8, pp. 4580-4610, 2017. 
[5] N. Islami, A. K. Ariffin, and S. Fonna, "Jurnal Teknologi The Effect Of Immersion Time On The Corrosion Behavior Of Sus304 In Brine," no. June, 2016.

[6] P. R. Roberge, Handbook of corrosion engineering. McGraw-Hill, 2000.

[7] M. A. Gebril, M. S. Aldlemey, and A. F. Kablan, Effect of tempering on mechanical properties and corrosion rate of medium and high carbon steel, vol. 685. 2013.

[8] J. Blanco, Y. Salas, C. Jiménez, Y. Pineda, and A. Bustamante, "Evaluation of the electrical conductivity and corrosion resistance for layers deposited via sputtering on stainless steel," in Journal of Physics: Conference Series, 2017, vol. 935, no. 1, p. 12017.

[9] V. Ashworth and C. J. L. Booker, "Cathodic protection: Theory and practice," 1986.

[10] E. S. Cavaco, A. Bastos, and F. Santos, "Effects of corrosion on the behaviour of precast concrete floor systems," Constr. Build. Mater., vol. 145, pp. 411-418, 2017.

[11] K. Shi et al., "Corrosion Behavior and Conductivity of TiNb and TiNbN Coated Steel for Metallic Bipolar Plates," Appl. Sci., vol. 9, no. 12, p. 2568, 2019.

[12] G. S. Frankel, "Fundamentals of corrosion kinetics," in Active protective coatings, Springer, 2016, pp. 17-32.

[13] S. Fonna, I. M. Ibrahim, M. Ridha, S. Huzni, and A. K. Ariffin, "Simulation of the ill-posed problem of reinforced concrete corrosion detection using boundary element method," Int. J. Corros., vol. 2016, 2016.

[14] C. A. Brebbia, J. C. F. Telles, and L. C. Wrobel, Boundary element techniques: theory and applications in engineering. Springer Science \& Business Media, 2012.

[15] J. T. Katsikadelis, The boundary element method for engineers and scientists: theory and applications. Academic Press, 2016.

[16] S. Fonna, S. Huzni, A. K. Ariffin, and others, "Boundary element inverse analysis for rebar corrosion detection: Study on the 2004 tsunami-affected structure in Aceh," Case Stud. Constr. Mater., vol. 8, pp. 292-298, 2018.

[17] H. L. Oliveira, A. Chateauneuf, and E. D. Leonel, "Boundary element method applied to decision-making problems involving geometric variabilities in topology optimization,” Eng. Anal. Bound. Elem., vol. 85, pp. 116-126, 2017.

[18] H. Koguchi and H. Watabe, "Improving defects search in structure by boundary element and genetic algorithm scan method," Eng. Anal. Bound. Elem., vol. 19, no. 2, pp. 105-116, 1997.

[19] A. BEASY, "Boundary Element Analysis System," C. Southampton, UK, Boston, USA, vol. 22, 1995.

[20] S. Fonna, S. Huzni, A. Zaim, and B. Aceh, "Simulation of Cathodic Protection on Reinforced Concrete Using BEM," vol. 4, no. 2, pp. 111-122, 2017.

[21] S. Aoki and K. Kishimoto, "Prediction of galvanic corrosion rates by the boundary element method," Math. Comput. Model., vol. 15, no. 3-5, pp. 11-22, 1991.

[22] K. Amaya, J. Togashi, and S. Aoki, "Inverse analysis of galvanic corrosion using fuzzy a priori information," JSME Int. journal. Ser. A, Mech. Mater. Eng., vol. 38, no. 4, pp. 541-546, 1995. 